Innenfläche mit weissgelblichen markigen Massen infiltrirt, die leistenartig in die Höhle prominirten. Die hintere Wand war inclusive der Cervicalpartie nur in 4-5 $\mathrm{Cm}$. Höhe erhalten, der Rest und der Fundus fehlten. Die zerrissenen Ränder dieses Defectes waren ebenfalls markig infiltrirt und sie adhärirten überall fest an dèr serösen Bekleidung der hinteren Beckenwand, also an der hinteren Wand des früheren Cav. Dougl. In diese so gebildete Geschwürshöhle führte die oben erwähnte Oeffnung am oberen Uterusrande.

Die mikroskopische Untersuchung des Uterus (Prof. Cohnheim) ergab den exquisit carcinomatösen Charakter der Infiltration.

Dass die Perforation des earcinomatösen Kraters in die Bauchhöhle und die daraus resultirende Peritonitis durch meinen letzten Eingriff verursacht war, ist wohl zweifellos. Dass letzterer berechtigt war, ebenso wie die Diagnose auf Schleimhautsarcom der Uterinhöhle, auf welcher er basirte - brauche ich kaum zu begründen. Symptome und objectiver Befund liessen kaum eine andere Annahme zu.

\title{
Zur operativen Behandlung der Uterusmyome.
}

\author{
Von \\ Dr. P. Müller, \\ Privatdocent zu Würzburg. \\ (Mit 2 Holzschnitten.)
}

Jüngst hat Spiegelberg in diesem Archive ${ }^{1}$ ) die Aufmerksamkeit auf eine gewisse Art der fibro-myomatösen Uterus-Geschïlste gelenkt, welche er als "eingekeilte" bezeichnet, irreponible Tumoren, die von den Wandungen des Uterus aus in die Scheide hereinwachsen, den Beckenkanal mehr oder weniger ausfüllen und hauptsächlich wegen der Druckerscheinungen, die sie hier setzen, ein therapeutisches Einschreiten erfordern.

Mit Recht hat er $\mathrm{Kiwisch}$ gegenüber, der eine symptomatische Behandlung für hinreichend erachtet, darauf hingewiesen, dass der das Leben bedrohende auf die Beckeneingeweide ausgeübte Druck

1) Band V. S. 100. 
der Geschwulst nur durch eine, wenn auch nur partielle Abtragung: der letzteren beseitigt werden könne.

Spiegelberg veröffentlicht bei dieser Gelegenheit zwei Fälle. wo dieser Anschauung gemäss eine Amputation des Tumors ausgefiihrt wurde. Allerdings sind diese Fälle keineswegs ermunternd, indem bei Beiden der Tod in Folge von Verjauchung und Septicaemie eintrat; aber fragen wir, was ist das Schicksal der Frauen, bei denen nicht in dieser Weise vorgegangen wird? Kann die Reposition nicht ausgeführt werden, so muss nach einem kurzen oder längeren qualvollen Siechthum, bei der Unmöglichkeit, dem Wachsthume des 'Tumors Einhalt zu thun, durch die Aufhebung der Functionen der Beckenorgane der lethale Ausgang sicher eintreten. Unter solchen Umständen ist gewiss ein operativer Eingriff ebenso gereehtfertigt, wie z. B. bei Ovariotomie, wenn dieselbe auch in Hinblick auf die Möglichkeit eines ungünstigen Ausganges unternommen würde.

Doch nicht inmer ist die Prognose eine so ungünstige, wie es bei einer ausschliesslichen Berücksichtigung der Spiegelberg'schen Fälle oder der von Sims') mit tödtlichem Ausgange ausgeführten ähnlichen Operationen den Anschein haben könnte; einige Fälle wenigstens, die ich. schon vor der Spiegel berg'schen Publication aus dem nämlichen Grunde einer operativen Behandlung unterzog, dürften wohl zeigen, dass solche Amputationen ohne ungünstige Folge ausgeführt werden können, und dass sie ibren Zweek vollkommen zu erreichen im Stande sind. Gewissermassen zur Ergänzung des Spiegelberg'schen Aufsatzes erlaube ich mir diesen casuistischen Beitrag zu liefern.

1) B. Ls., Lehrerstochter, 38 Jahre alt, ledig, litt als Kind an Scrophulose. In der Pubertät traten chlorotische Erscheinungen auf, die durch mehrere Jahre anhielten. Die Menstruation trat erst nach dem 20. Lebensjahre ein; sie war im höchsten Grade unregelmässig, oft längere Zeit aussetzend, oft als starke Blutungen sich zeigend. Geboren hat die Patientin nie. Mit dem Jahre 1867 wurde die Menstruation reichlicher und wiederholte sich öfter als früher. Starker Fluor albus stellte sich ein. Erst im Jahre 1871 wurde ärztliche Hülfe gesucht, als die Beschwerden bei der Urinentleerung heftiger wurden, welche öfters die Anlegung des Katheters nothwendig machten. Dabei wurde die Patientin von hartnixckiger Stuhlverstopfung gequält, die nur durch starke Abführmittel gehoben werden konnten. Der Gang wurde unsicher, eine starke Abmagerung trat ein; Mitte März 1871 wurde ich von dem behandelnden Arzte Dr. Millberger zugezogen.

Befund: Kleine Figur, sehr abgemagert, starke Augenentzündung, an verschiedenen Stellen Narben von scrophulösen Geschwüren. Bei der äusseren Untersuchung fand ich neben der Sym-

1) Klinik der Gebärmutterchirurgie. Beigel'sche Uebersetzung. S. 88. 
physe mehr nach links einen gänseeigrossen Tumor. Vor den äusseren Genitalien lag eine über faustgrosse Geschwulst, die wie ein Kindeskopf bei der Geburt die Schamspalte ausdehnte und die sich ohne Unterbrechung in die Scheide fortsetzte, das gange Becken ausfibllend. Die innere Untersuchung konnte nur in der Chloroformnarkose vorgenommen werden und war nur durch den Umstand möglich, dass der Tumor zu den weichen Fibroiden gehörte. Sowohl im hinteren Scheidengewölbe als seitlich war nichts von einem Muttermunde zu entdecken, nur vorn, hinter der Symphyse war die vordere Muttermundslippe als dünner, $3 \mathrm{Cm}$. langer, scharfer Saum zu entdecken, hinter welchem und vor dem Tumor man mit der Sonde auf höchstens $3 \mathrm{Cm}$. in die Höhe dringen konnte; demnach sass der Tumor mit einer breiten, Basis auf der hinteren Cervical-Uteruswand und zugleich an dem Fundus der Gebärmutter auf. Eine eigentliche Höhle des Corpus uteri schien nicht mehr zu existiren.

Wegen der heftigen Blutungen, besonders aber wegen der Druckerscheinungen schritt ich zur Amputation des in die Scheide herunterragenden Theiles der Geschwulst. Letztere liess sich etwas mit der Muzeaux' schen Zange herunterziehen, so dass ich dann, unterstützt durch die Weichheit des Tumors, die Kette des Ecraseur hoch in das hintere Scheidengewölbe und vorn am Muttermunde anlegen konnte. Aber trotzdem ich eine halbe Stinde hindurch mit grossem Kraftaufwande an der Kurbel drehte, wurde der Tumor nicht abgetrennt; er. wurde durch die Kette nur hochgradig zusammengequetscht, so dass ich hart unter der Kette die Geschwulst mit der Scheere abtrug. Nach Entfernung derselben zeigte sich, dass der Amputationsstimpf den ganzen Beckeneingang ausfüllte; noch deutlicher als früher konnte vorn an dernselben eine kaum $3 \mathrm{Cm}$. tiefe Tasche constatirt werden, die den Ueberrest der Uterushöhle darstellte.

Der abgetragene Theil wurde von Dr. Köster untersucht und für eine fibromyomatöse Neubildung erklärt. Auffallend rasch verkleinerte sich der Uterus mit seinem Amputationsstumpfe; letzterer wurde immer kleiner, so dass er nach einigen Wochen eine hochrothe, kaum thalergrosse Fläche darstellte. Der über der Symphyse fühlbare Tumor schwand jedoch nicht. Die Kranke erholte sich sehr rasch, so dass schon nach kurzer Zeit von der früheren Abmagerung nichts mehr zu bemerken war. Die Menstruation wurde regelmässig, wenn auch stark; Druckerscheinungen im Becken wurden nicht mehr wahrgenommen. Der Gebrauch der Extremitäten war ein ganz normaler. Dieser günstige Gesundheitszustand dauerte gerade ein Jahr; da traten neue Blutungen ein, und bei der inneren Untersuchung fand ich in der Scheide einen etwa $6 \mathrm{Cm}$. langen, dünnen, walzenförmigen, leicht blutenden Körper, der von dem früheren Amputationsstumpfe ausging. Der Tumor über der Symphyse war beträchtlich gewachsen. Ich trug Mitte April 1872 diese Excrescenz ab; dieselbe erwies sich als ein sarcomatoeses Neoplasma. Die Patientin erholte sich 
nicht mehr: neue Blutungen traten ein, die Excrescenz wuchs bis zum August desselben- Jahres zu der früheren Grösse heran, so dass in meiner Abwesenheit von Würzburg Dr. Millberger am 5. August dieselbe abermals mit der Seheere entfernte. Unter geringen peritonitischen Erscheinungen erfolgte der Tod am 10. Aug.

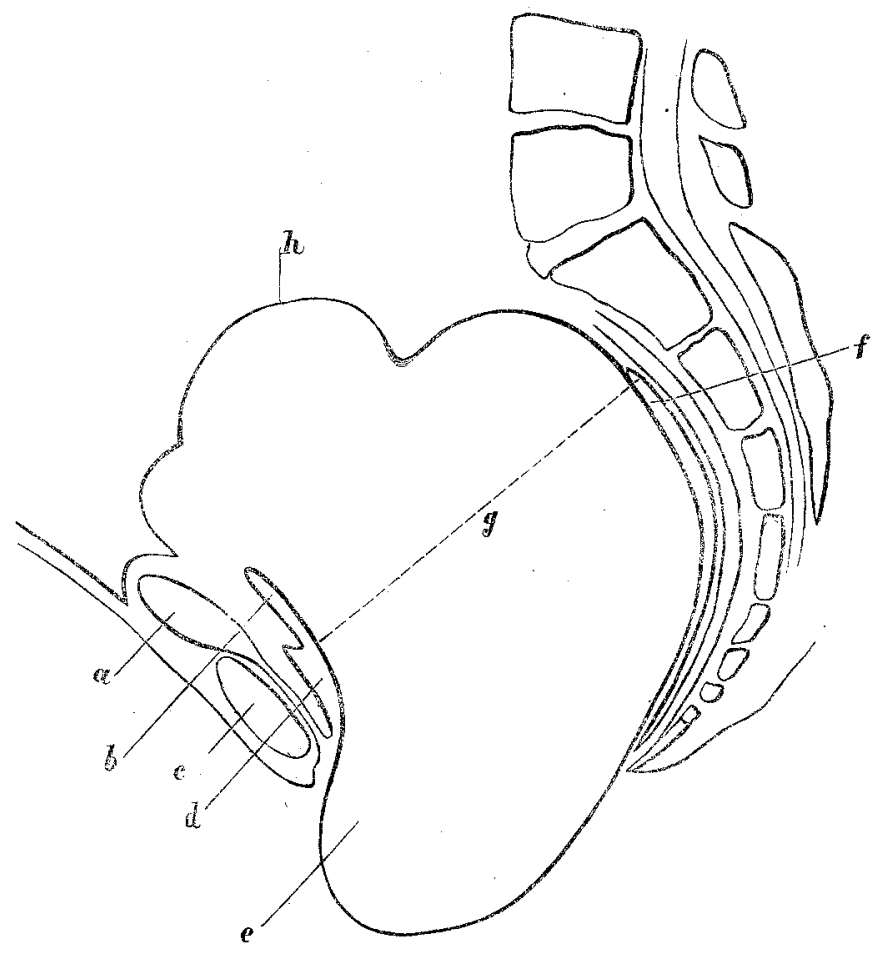

Die Section, von Dr. Köster ausgeführt, ergab Folgendes: Induration in der Lungenspitze, Lungenoedem, allgemeine eitrige Peritonitis. Sehr grossen sarcomatösen Tumor an der hinteren Wand des Uterus aufsitzend und mit der Vagina verwachsen. Sarcomknoten in dem Ligamentum latum. Secundäre Knötchen in der Leber. - Es scheint in dem zurückgebliebenen Theile des Tumor nach der Operation eine sarcomatöse Umwandlung, welche zu bedeutenderem Wachsthume und zur Bildung der Excrescenzen Veranlassung gab, eingetreten zu sein.

Figur. 1.

a. Blase. b. Ueberrest der Uterushöhle. c. Symphyse. d. Vorderes Scheidengewölbe mit Muttermund. e. Abgetragene Partie des Tumors. f. Hinteres Scheidegewölbe. g. Amputationslinie. $h$. Kleiner im Fundus uteri sitzender Knoten. 
2) L, B., Lehrersfrau von R., jetzt 47 Jahre alt, war als Kind gesund; mit dem 13. Jahre trat die Menstruation ein; die Menses waren stark, mit Schmexzen verbunden. Chlorotische Erscheinungen in der Pubertät. Mit dem 22. Jahre verheirathet, gebar dieselbe im 23., 26. und 35. Lebensjahre regelmässig; die Menstruation ging

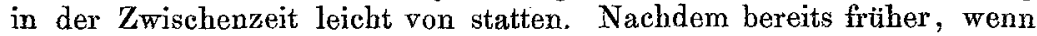
auch nur vorübergehend, Störungeñ der Menstruation eingetreten waren, wurden vom Jahre 1867 an die katameniale Blutungen selir profus, lange dauernd, später nur einen kurzen Raum von wenigen Tagen zwischen sich lassend. Heftige wehenartige Schmerzen und eine profuse Secretion bewirkten einen starken Kräfteverfall; dazu traten dann Mitte des Jahres 1871 heftige Blasenbeschwerden, so dass öfter der Katheter angelegt werden musste; hartnäckige Stublverstopfungen erforderten die Anwendung von Drastica. Besonders belästigend waren Hämorrhoidalbeschwerden; starke Varicen bildeten sich an den unteren Extremitäten aus, starke Oedeme an den nämlichen Theilen stellten sich zeitweise ein. Dieser Zustand veranlasste die Kranke September 1871 bei mir Hülfe zui suchen. Bei der äusseren Untersuchung fand ich einen Tumor, der 2 Finger breit über die Symphyse hervorragte, dabei glatt und eben; bei der inneren Untersuchung stiess ich in der Mitte der Vagina auf eine runde Geschwulst, die das kleine Becken fast vollständig ausfüllte. Nur mit Mühe liess sich der Finger zwischen der Geschwulst und der Vagina hinauffübren. Im vorderen Scheidengewölbe war der Muttermund zu fühlen, die vordere Lippe an der Symphyse anliegend, war in die Breite gezogen and bildete einen scharfen Rand; die hintere Lippe war ganz in die Geschwulst aufgegangen; das Hintergewölbe war sehr schwer zu erreichen. Die Sonde drang iiber $8 \mathrm{Cm}$. tief mit nach hinten gerichteter Spitze ein.

Die von der Patientin dringend gewünschte Operation wurde Mitte September bei Anwesenheit der Herren DDr. Osann und Reuss ausgeführt. Ich versuchte zuerst die in die Scheide hineinragende Hälfte des Tumors mittels der galvanocaustischen Schlinge abzutragen. Allein der Apparat versagte mitten in der Arbeit seinen Dienst, so dass ich die Amputation mittels der Scheere vollenden musste. Mein Plan war ursprünglich, nach der Amputation des untereren Stückes die obere Hälfte auszuschälen; allein eine Kapsel der Geschwulst existirte nicht; die Enucleation war deshalb unmöglich. Eine weitere Abtragung des Stïckes mittels Zerstückelung unterliess ich, da ich vorerst die Veränderung am Amputationsstumpfe beobachten wollte. Es wäre ja möglich, dass der Rest durch Eiterung abgestossen oder durch Schrumpfung derselbe wesentlich verkleinert würde. Die erste Möglichkeit trat nicht ein, dagegen wurde unter starker Eiterung der Ueberrest des Tumors etwas kleiner, aber keineswegs war die Verringerung des Volumens so bedeutend, als ich es nach den Beobachtungen Braun's über die Verkleinerung des Uterus vach Abtragung der Vaginalportion erwartet hätte. Aber ihren $Z$ weck hat die Operation doch erreicht. Ich will die Zeit der Nachbehandlung hier ganz 
iibergeben und nur bemerken, dass bereits nach kurzer Zeit die Patientin sich vollständig erholt hatte und überhaupt alle Beschwerden geschwanden waren. Jetzt, zwei Jahre nach der Operation, habe ich die Frau einer Untersuchung unterzogen. Der Ueberrest der Geschwulst ist noch vorhanden, die Verkleinerung eine geringe, der Amputationsstumpf vernarbt, bis auf wenige inselförmige Stellen. Aber was die Hauptsache ist, das Befinden der Frau ist ein ausgezeichnetes. Die Menstruation tritt regelmässig alle 3-4 Wochen

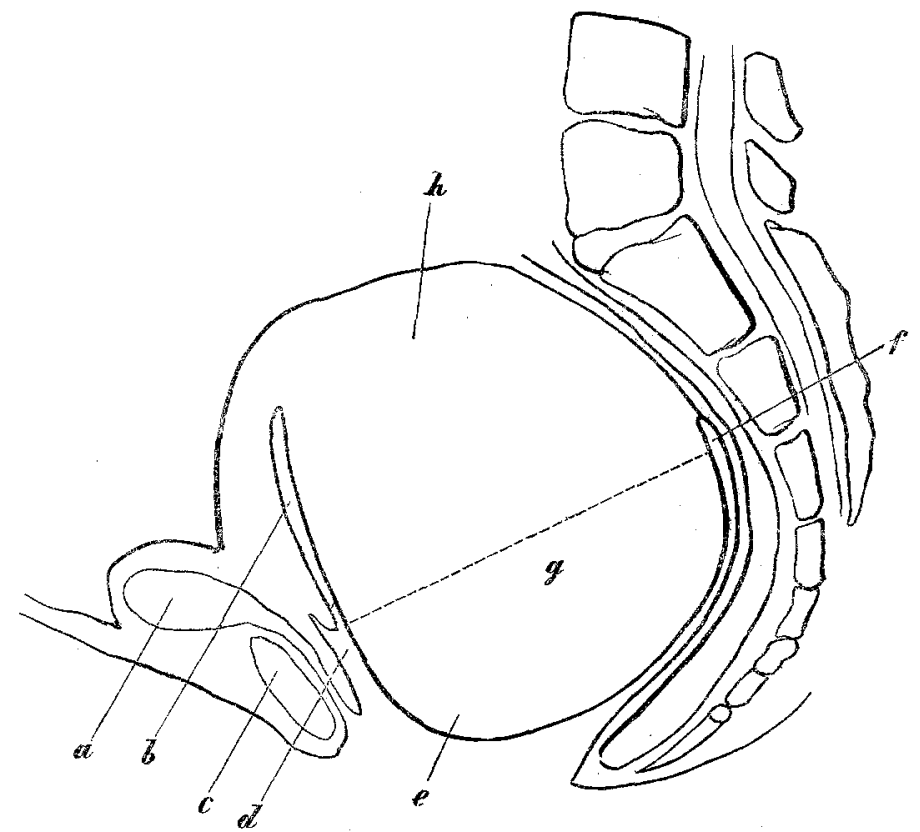

ein, dauert zwar 8 Tage, aber nur 1 bis 2 Tage reichlich, dann spärlich ohne Schmerzen; die Urinansscheidung regelmässig, ohne Beschwerden. Der Stuhl eher zu Diarrhöen als zur Verstopfung geneigt. Die Hämorrhoidalbeschwerden gering. Die Ansehwellungen der Extremitäten sehr selten auftretend und dann nur auf den Fuss sich beschränkend. Die unteren Extremitäten leicht beweglich, das Gehen nicht mehr erschwert. Die Auftreibung des Leibes gering, das Aussehen ein sehr gutes.

Figur II.

a. Blase. b. Uterinhöhle. e. Symphyse. d. Vorderes Scheidengewölbe mit Os extern. ut. e. Abgetragene Partie des Tumors. $f$. Hinteres Scheidengewölbe. $g$. Amputationslinie. $h$. Zurückgebliebene Partie der Geschwulst. 
In diesen beiden Fällen, wo doch gewaltige Schnittflächen durch die Amputation gebildet wurden, sind keine accidentellen $W$ undkrankheiten hinzugetreten; im ersten Falle, trotz der Anwesenheit des zweiten Tumors im Uteruskörper innerhalb wenige Wochen der Amputationstumpf auf ein Minimum reducirt, während im zweiten, wo die Hälfte des Tumors sitzen blieb, die Schnittflïche relativ rasch vernarbte. Ueberraschend war in beiden Fällen der Heileffect. Bei der ersten Patientin wurde auf die Dauer eines Jahres eine höchst zufriedenstellende Besserung erzielt, welche gewiss noch länger angedauert hätte, wenn nicht im Amputationsstumpf die sareomatöse Neubildung aufgetreten wäre, während die zweite Patientin jetzt, nach 2 Jahren, trotz der Gegenwart der zurückgebliebenen Reste eines ausgezeichneten Wohlbefindens sich erfreut.

Nur um zu zeigen, wie leicht manchmal die Abtragung solcher in die Vagina hineinragenden fibromyomatösen Geschwiulste vertragen wird, möchte ich noch auf einen Fall hinweisen, den ich vor einigen Jahren in einem Aufsatze ${ }^{1}$ ) beschrieben, in welchem ich die vom unteren Uterussegment ausgehenden Fibroide einer Besprechung unterzog. In diesem Falle trug ich mittels der galvanocaustischen Schlinge ein von der hinteren Cervicalwand ausgehendes Myom ab, das freilich noch nicht bedenkliche Druckerscheinungen verursacht hatte. Obgleich die Schnittfläche $10 \mathrm{Cm}$. in der einen und $7 \mathrm{Cm}$. in der anderen Dimension betrug, verlief die Nachbehandlung ohne alle Störung.

Aber nicht blos der Druck auf die Beckenorgane wird durch die Abtragung beseitigt, sondern der letzte Eingriff scheint nicht ohne günstigen Einfluss auf den zurïckgelassenen Ueberrest des Tumors zu sein. An und für sich sollte man annehmen, dass bei dem Umstande, dass noch ein grosser Theil der Neubildung zurückbleibt, der ja eines weiteren Wachsthums fähig ist, die Blennorrhoe, Blutungen, Uterinalcoliken nach wie vor fortdauern müssten, allein in unseren beiden Fällen wurde auch hier eine wesentliche Besserung erzielt. Wie geradezu indicirt es ist, bei Uterustumoren, welche in den Wandungen des Uterus sitzen und keiner totalen Abtragung fähig sind, Alles zu entfernen, was nor von der Scheide aus zugängig ist, beweist mir folgender Fall, den ich bier nur kurz erwähnen will.

Frau F. aus Landan, 40 Jahre alt, litt seit einer langen Reihe von Jahren an einem Fibromyom des Uterus. Symptome dieser Neubildung waren seit Jahren vorhanden. In dem letzten Jahre, ehe ich die Patientin sah, entwickelte sich ziemlich rasch eine bedeutende Hypertrophie der Vaginalportion, welche Letztere von etwa Gänseeigrössse den oberen Theil der Scheide ausfüllte. Die Patientin war durch die profusen Blutungen und Blennorrhoeen hochgradig anämisch, an Bett und Zimmer gefesselt. Ich trug um Ende August 1871 in Gemeinsehaft mit den Herren DDr. Hitschler, Eichborn sen.

1) Scanzoni's Beiträge, Band VI. S. 67. 
und jun. mittels der galvanocanstischen Schlinge die Vaginalportion ab. Auch hier war der Erfolg, trotzdem das Fibroid vollständig intact blieb, ein höchst zufriedenstellender. Auch jetzt, nach 2 Jahren, ist der Gesundheitszustand nach den mir zugehenden Berichten, gegenüber der früheren Zeit, ein sehr günstiger.

\title{
Zum Verhalten des Scheideneinganges nach der Geburt.
}

\author{
Vorläufige Mittheilang \\ von
}

E. Bellien, stud. med, in Charkow.

Bei den Untersuchungen des Verhaltens des Hymen in seinen verschiedenen physiologischen Zuständen, respective des Verhaltens seiner Reste nach der Geburt, welche ein Material von 286 Exemplaren betreffen, die ich in hiesiger geburtshülflichen Klinik auszuführen die Möglichkeit hatte, gelangte ich zu Resultaten, welche mir der Mittheilung nicht unwerth erscheinen; dieselben sind theils Bestätigung des schon Bekannten, theils keziehen sie sich auf neuergründete Thatsachen - die Entstehungsweise der Carunculae myrtiformes betreffend.

Vorliegende Mittheilung betrifft nur die Veränderungen des $\mathrm{Hy}_{y}$ men nach der Geburt.

Sehröder in Erlangen hat in letzterer Zeit mehrere Male darauf aufmerksam gemacht (in ,Sehwangerschaft, Geburt und Wochenbett". 1867. \$. 6., in der Gynäkologischen Section der Versammlung dentseher Naturforscher und Aerzte in Rostock. 1871. Sitzung 18. September), dass die bisherigen Vorstellungen vom Hymen und den Carunculae martiformes nieht ganz zutreffend sind. Seiner Meinung nach werden die Carunculae myrtiformes nie vor der ersten Geburt beobachtet; ihre Entstehung verdanken dieselben einer blutigen Suffundirung und späteren im Wochenbette eintretenden Gangränescenz der einzelnen Lappen des Hymen, welche dureh den Druck, dem dieselben beim Durchschneiden des Kindskopfes ausgesetzt sind, bedingt wird. Meine Beobachtungen erlauben mir die Richtigkeit der ersten Meinung vollständig zu constatiren; nie habe 